\title{
Microcrystal Electron Diffraction for Molecular Design of Functional Non-Fullerene Acceptor Structures
}

\author{
Steve Halaby, ${ }^{a}$ Michael W. Martynowycz, ${ }^{\mathrm{a}}$ Ziyue Zhu, ${ }^{\mathrm{b}}$ Sergei Tretiak, ${ }^{\mathrm{c}, \mathrm{d}}$ Andriy \\ Zhugayevych, ${ }^{\mathrm{d} *}$ Tamir Gonen, ${ }^{\mathrm{a} *}$ Martin Seifrid ${ }^{\mathrm{b} *}$ \\ ${ }^{a}$ Howard Hughes Medical Institute, David Geffen School of Medicine, Departments of Biological \\ Chemistry and Physiology, University of California, Los Angeles, California 90095, United States \\ ${ }^{b}$ Center for Polymers and Organic Solids, Department of Chemistry and Biochemistry, University of \\ California, Santa Barbara, California 93106, United States \\ ${ }^{c}$ Physics and Chemistry of Materials, Theoretical Division and Center for Integrated Nanotechnologies, \\ Los Alamos National Laboratory, Los Alamos, New Mexico 87545, United States \\ ${ }^{d}$ Skolkovo Institute of Science and Technology, 143026, Moscow, Russia
}

Supplementary Information

Table of Contents

S1 Analysis of o-IDTBR MicroED structure …...................................................................... 2

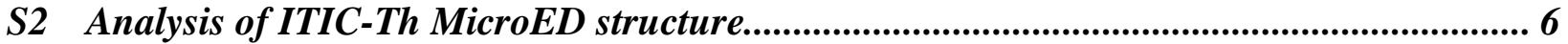

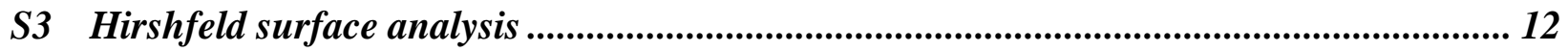

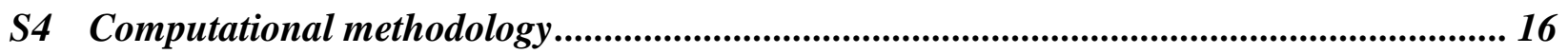

S5 Calculated structural properties .................................................................................... 18

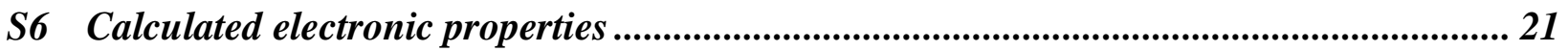




\section{S1 Analysis of o-IDTBR MicroED structure}
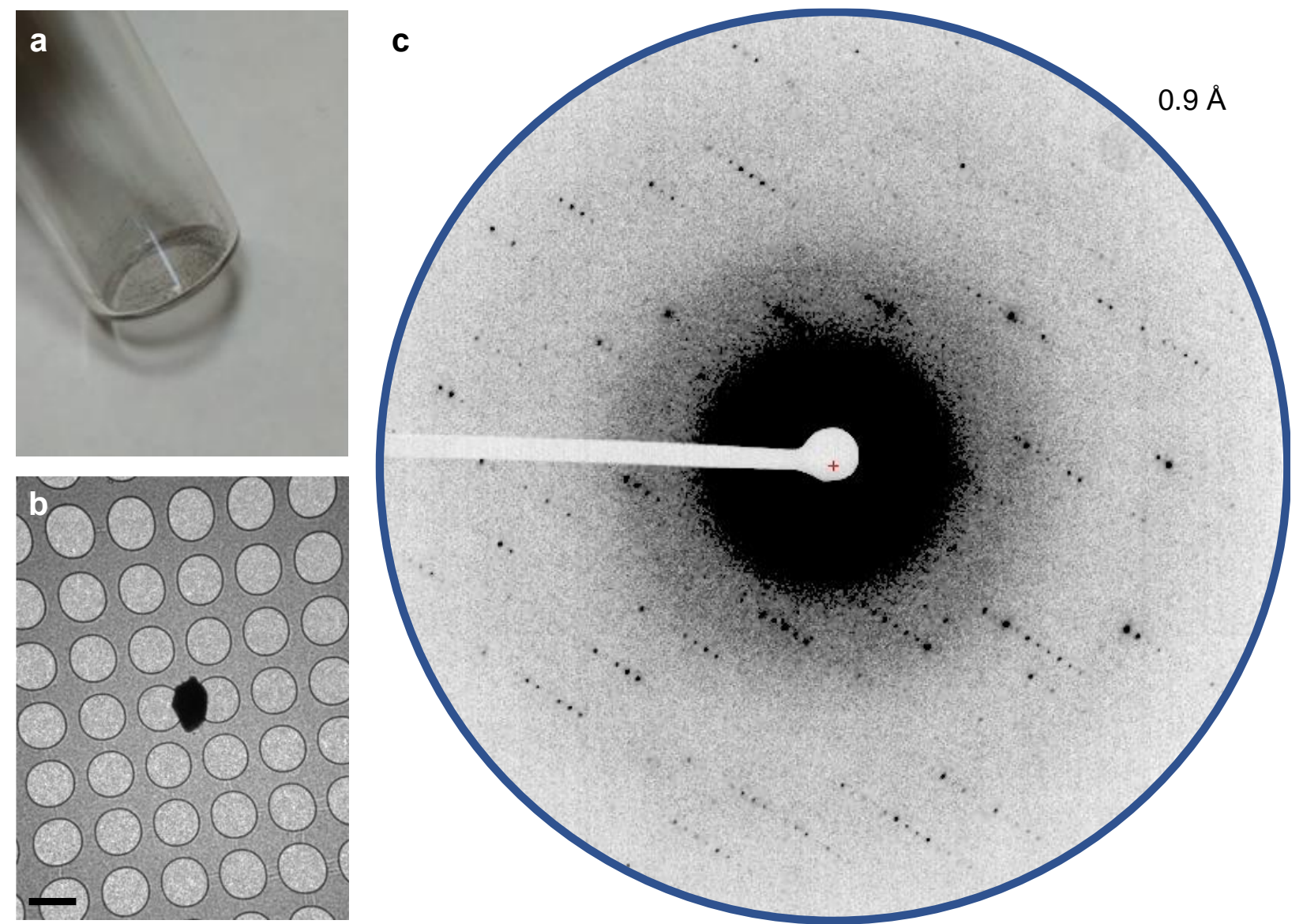

Figure S1. (a) Photograph of the o-IDTBR powder. (b) Image of the o-IDTBR crystal used for diffraction. The scale bar corresponds to $2 \mu \mathrm{m}$. (c) Diffraction image of o-IDTBR with resolution ring at $0.90 \AA$. 
Table S1. Data processing statistics of the MicroED structures for o-IDTBR and $\beta$-ITIC-Th.

\begin{tabular}{lll} 
& O-IDTBR & $\beta$-ITIC-Th \\
\hline Stoichiometric formula & $\mathrm{C}_{72} \mathrm{H}_{88} \mathrm{~N}_{6} \mathrm{O}_{2} \mathrm{~S}_{8}$ & $\mathrm{C}_{86} \mathrm{H}_{74} \mathrm{~N}_{4} \mathrm{O}_{2} \mathrm{~S}_{8}$ \\
Space group & $\mathrm{P} 2{ }_{1} / \mathrm{c}$ & $\mathrm{P} 2{ }_{1} / \mathrm{c}$ \\
Unit cell lengths $a, b, c(\AA)$ & $13.73,15.68,32.72$ & $15.32,29.00,15.46$ \\
Unit cell angles $\alpha, \beta,\left(^{\circ}\right)$ & $90.00,95.88,90.00$ & $90.00,97.49,90.00$ \\
Unique reflections (\#) & $7176(538)$ & $7143(548)$ \\
$\mathrm{R}_{\text {obs }}$ & $12.3(44.0)$ & $17.4(71.8)$ \\
$\mathrm{R}_{\text {meas }}$ & $14.9(77.9)$ & $19.7(81.2)$ \\
$\mathrm{CC}_{1 / 2}$ & $98.5(66.8)$ & $98.2(65.1)$ \\
Resolution $(\AA)$ & $10.3-0.9(0.92-0.90)$ & $15.19-1.0(1.03-1.00)$ \\
Completeness $(\%)$ & $71.3(70.1)$ & $95.6(96.9)$ \\
Total exposure $\left(\mathrm{e}^{-} \AA^{-2}\right)$ & $<1$ & $<1$ \\
$\mathrm{R}$ & 0.27 & 0.27 \\
wR2 & 0.52 & 0.52 \\
GooF & 2.31 & 2.76 \\
\hline
\end{tabular}

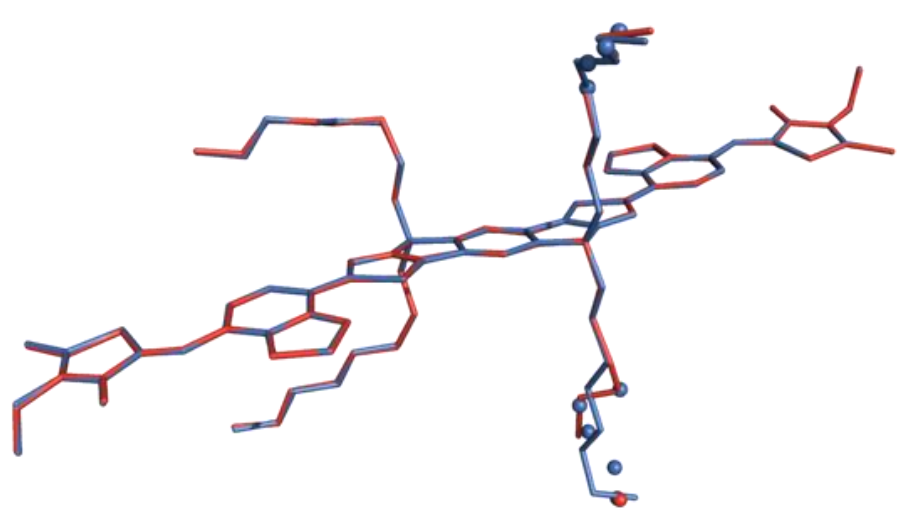

Figure S2. Overlaid structures of o-IDTBR determined by MicroED (red) and X-ray crystallography (blue). 
Analysis of the MicroED structure (Figure S3) shows that the backbone is very planar on the left side (L), with dihedral angles of $2.42^{\circ}$ between the indacenodithiophene (IDT) and benzothiadiazole (BT) moieties and $0.85^{\circ}$ between the BT and rhodanine (Rh) moieties; on the right side (R), the backbone is slightly twisted, with a $4.79^{\circ}$ IDT-BT dihedral and a $11.55^{\circ} \mathrm{BT}-\mathrm{Rh}$ dihedral. On the L side of the molecule, the $\mathrm{S}-\mathrm{N}$ distance is $2.95 \AA$, while it is $2.87 \AA$ on the $\mathrm{R}$ side. The rhomboid shape formed by four molecules has angles of $\sim 43^{\circ}$ and $\sim 139^{\circ}$, with a length of one molecule on each side. Each layer in the MicroED structure of o-IDTBR can be thought of as a wire mesh-type arrangement.

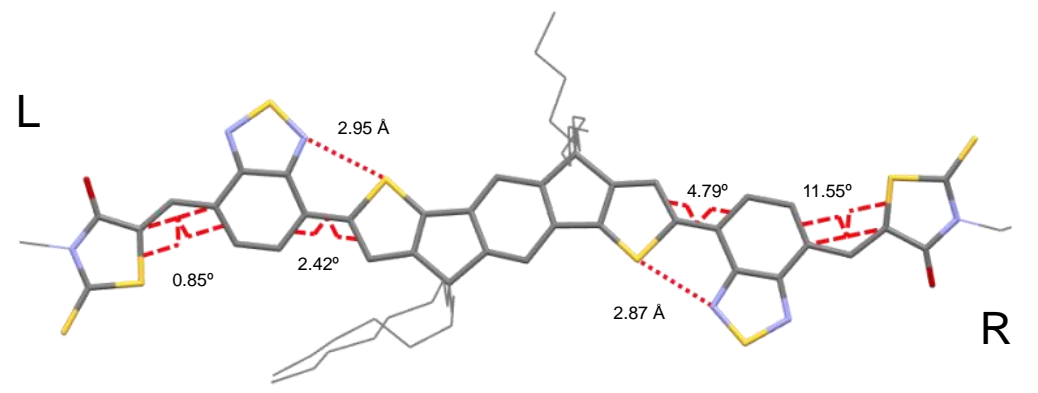

Figure S3. Dihedral twists and intramolecular $\mathrm{S} \cdots \mathrm{N}$ contact distances in the o-IDTBR MicroED structure.

The RR pair (Figure S4a) features $\pi \cdots \pi$ stacking between the BT units and intermolecular contacts between the Rh and indacenodithiophene (IDT) units. The stacking distance between the BT units is 3.26 $\AA$, and the distance between the Rh vinyl and carbonyl carbons and IDT sulfur is $3.39 \AA$ and $3.40 \AA$. There are also short intermolecular contacts between the Rh carbonyl oxygen and the hydrogens on the C4 $(2.27 \AA)$ carbons of the neighboring octyl chain. On the other hand, the intermolecular contact area in the LL pair (Figure S4b) is limited to overlap between the BT and Rh heterocycles. Analysis of short intermolecular contacts reveals $\pi \cdots \pi$ stacking distances between BT and Rh between $3.35 \AA$ and $3.56 \AA$. The LR/RL pair (Figure S4c) features two o-IDTBR molecules at $\sim 139^{\circ}$ relative to each other, with intermolecular short contacts between both BT units and between the Rh and IDT units. The BT units and methine carbon form a $\pi \cdots \pi$ stack, with intermolecular short contact distances of $3.36 \AA$ and $3.30 \AA$. There are also a number of $S \cdots \pi$ and $S \cdots O$ contacts. The sulfur of the IDT unit interacts with the thiocarbonyl carbon of the Rh ring at a distance of $3.41 \AA$, and the Rh carbonyl oxygen interacts with the sulfur atom in the BT heterocycles with a short contact distance of $3.26 \AA$. 
(a)

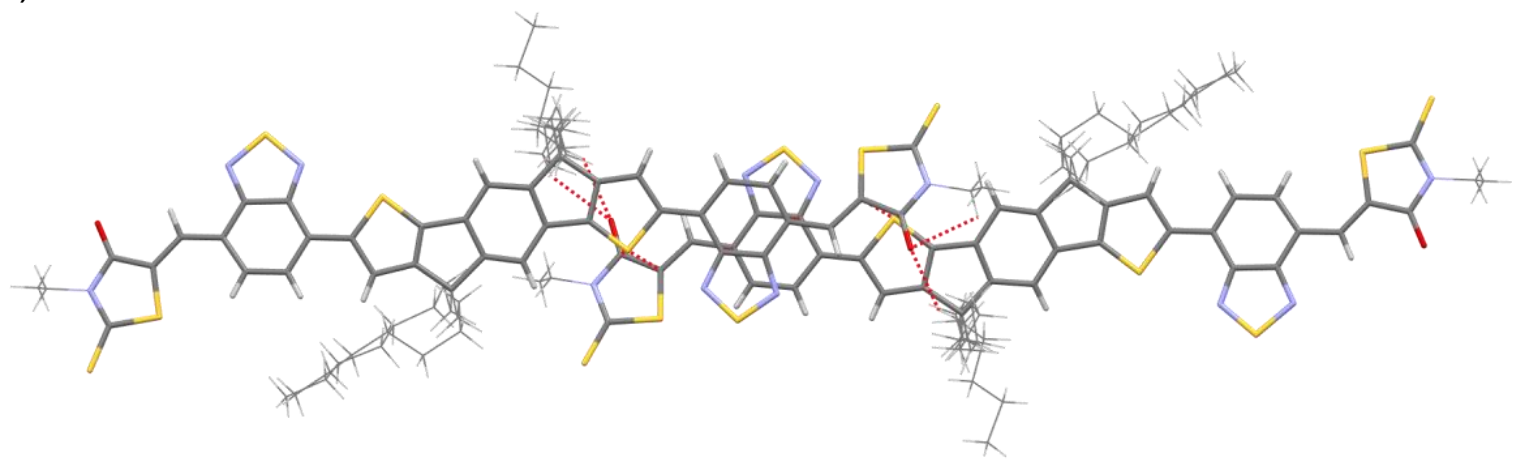

(b)

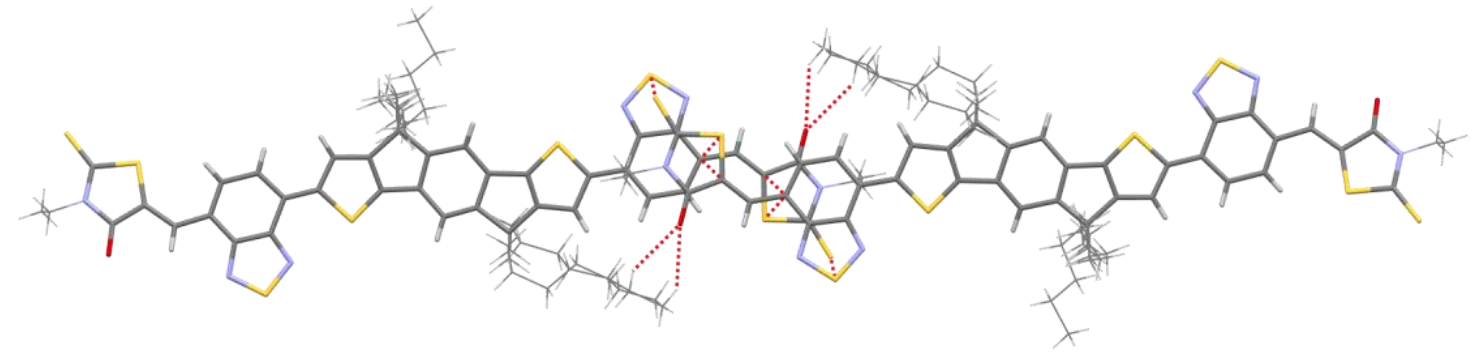

(c)

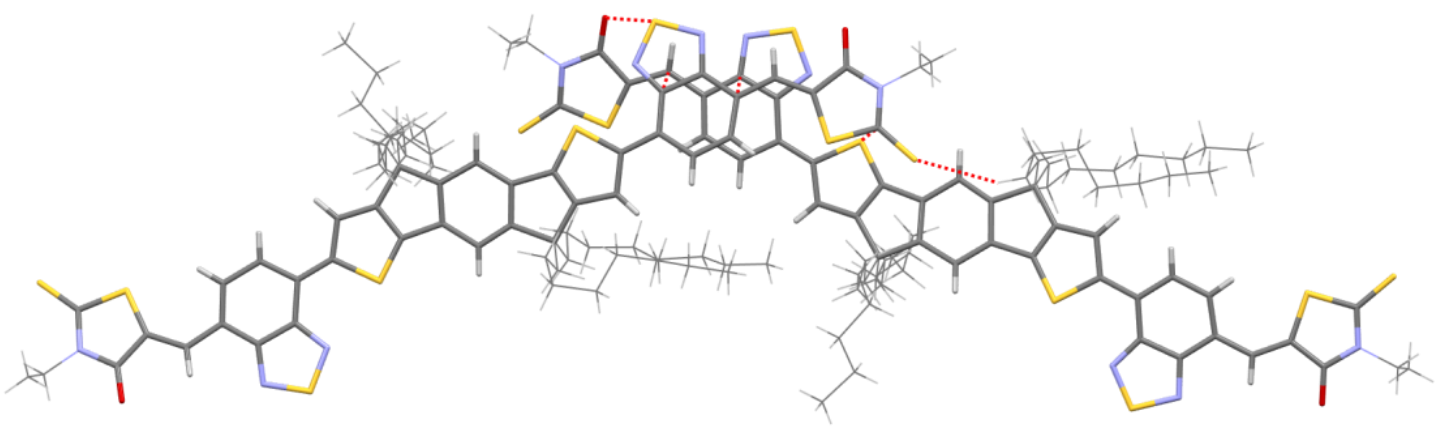

Figure S4. (a) Parallel close (RR), (b) parallel far (LL) and (c) obtuse (LR/RL) contacts in the o-IDTBR MicroED structure. 


\section{S2 Analysis of ITIC-Th MicroED structure}

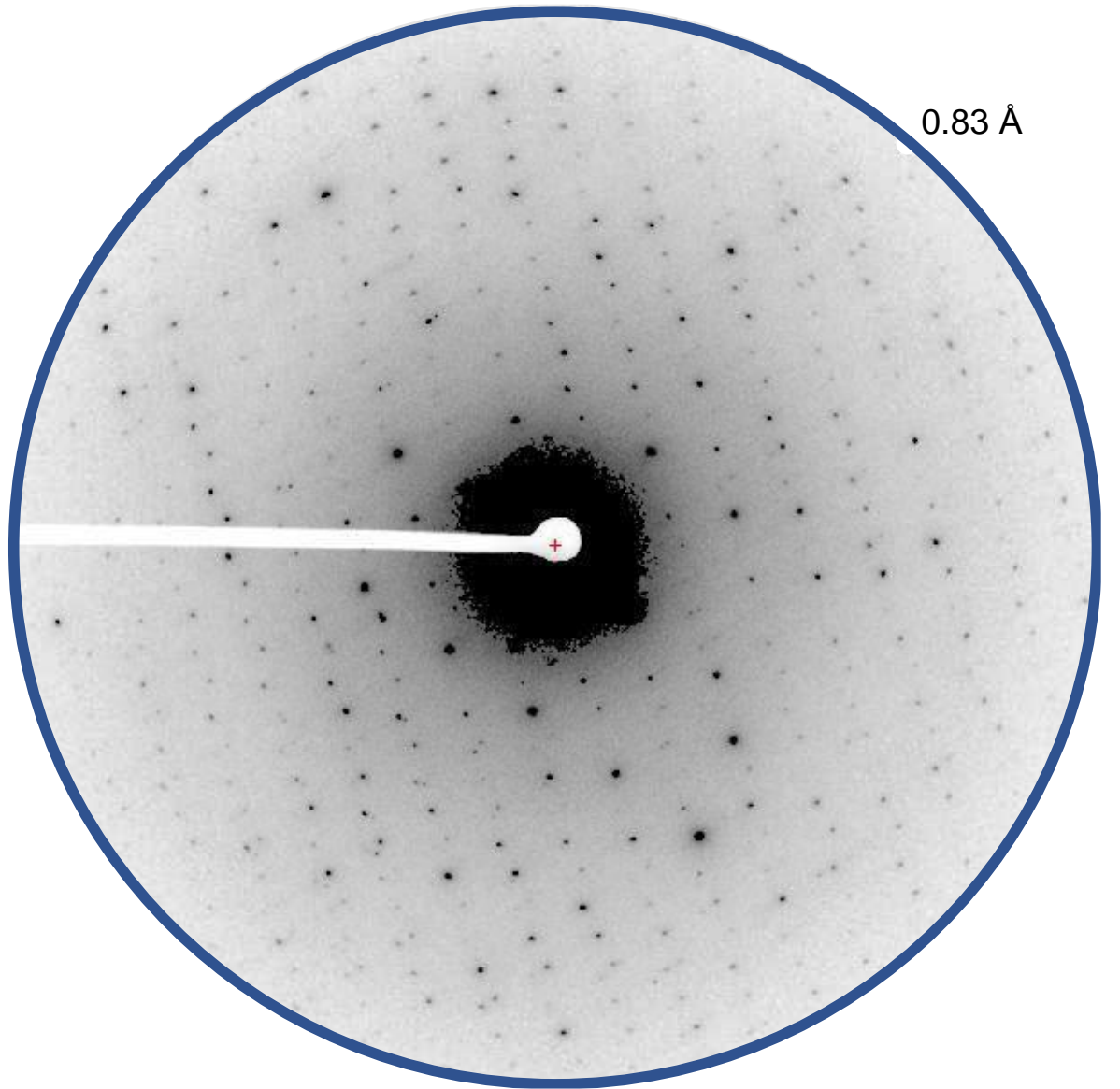

Figure S5. Diffraction image of $\beta$-ITIC-Th with resolution ring at $0.83 \AA$. 

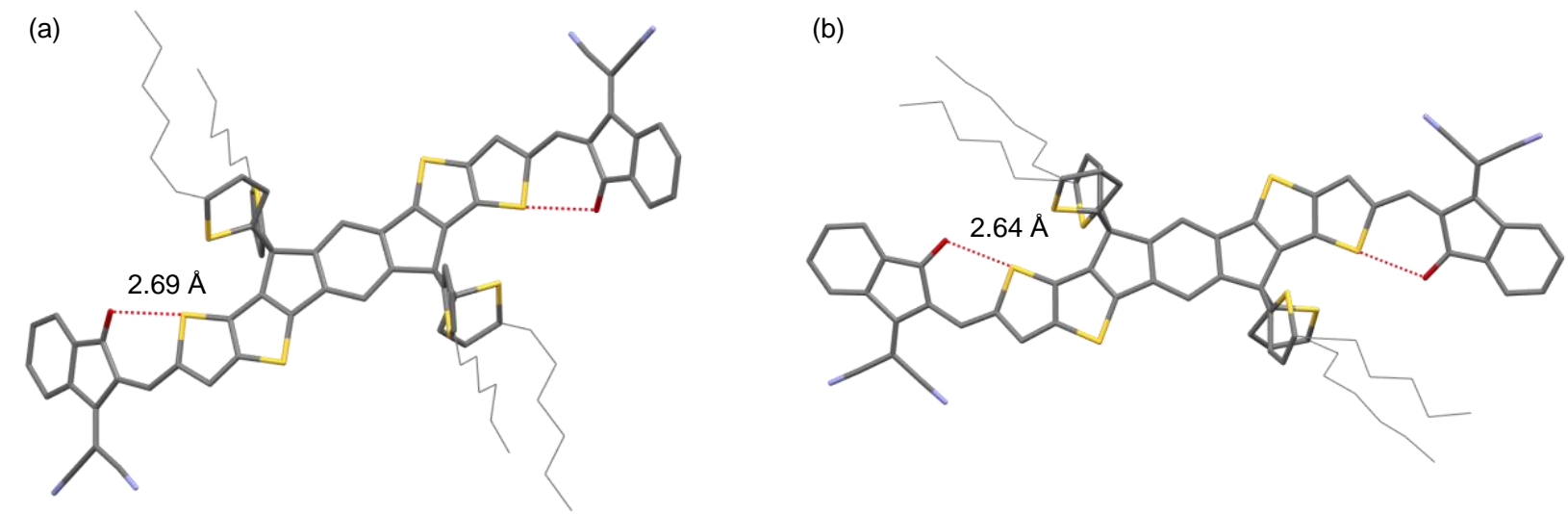

Figure S6. Intramolecular S $\cdots \mathrm{O}$ short contacts in the (a) HC and (b) LC conformers of the $\beta$-ITIC-Th MicroED structure. 
(a)

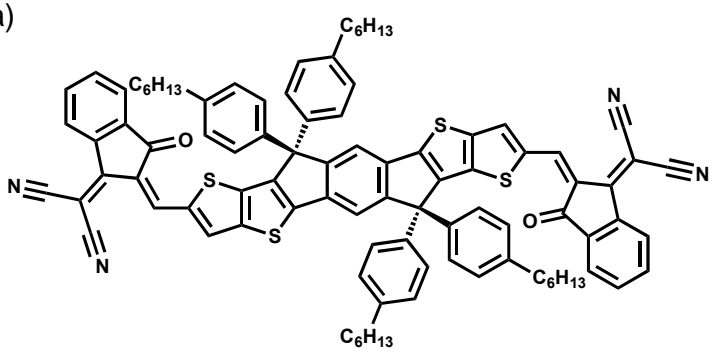

(c)

(e)
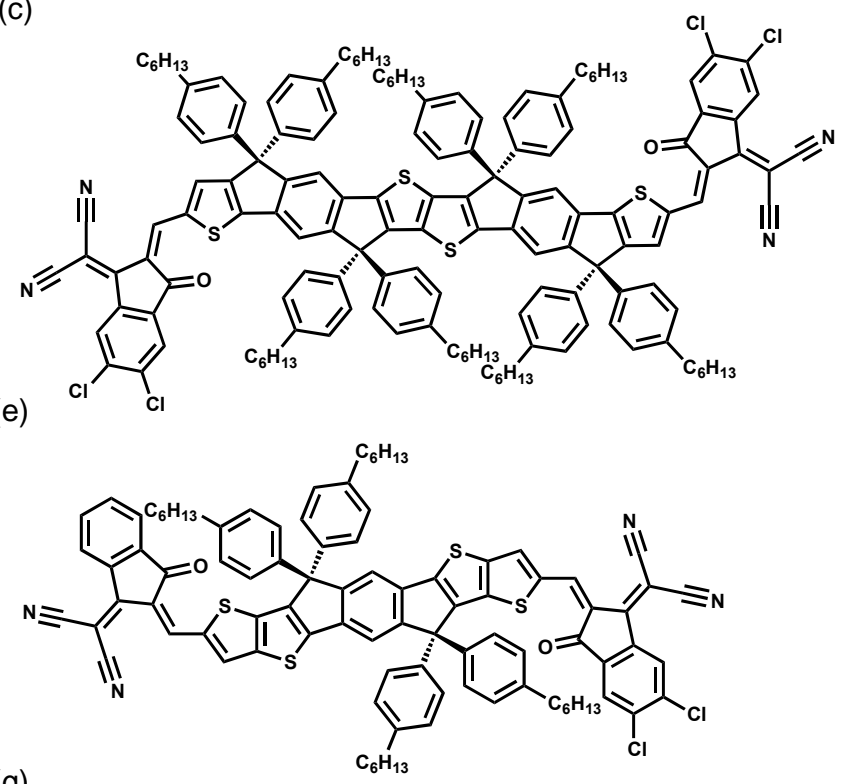

(g)

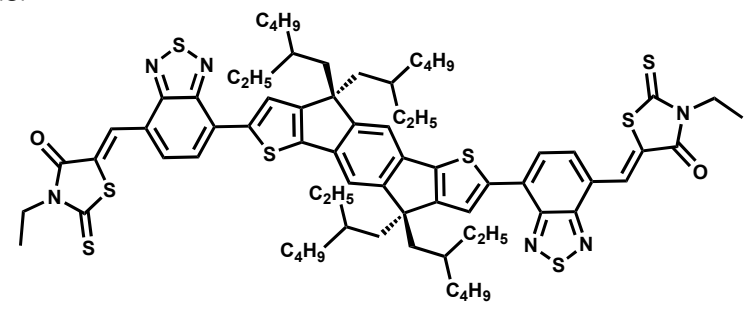

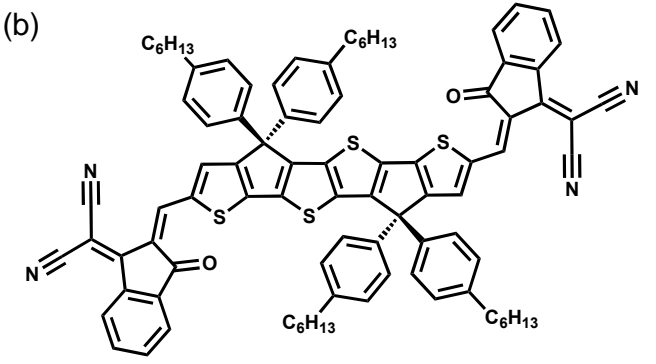

(d)

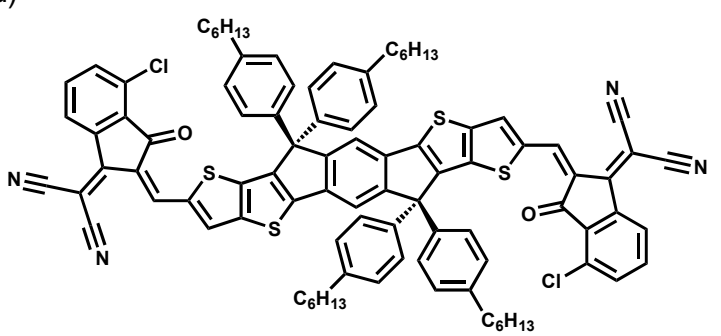

(f)

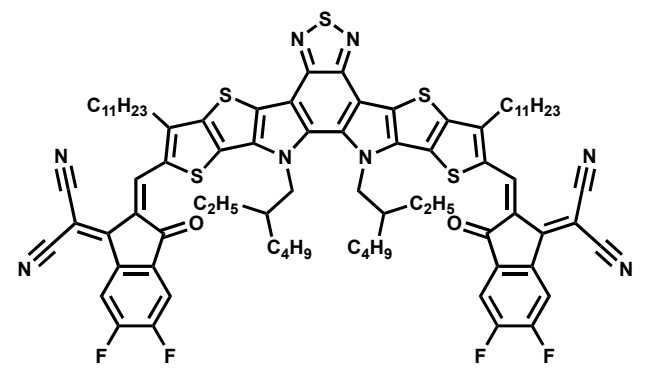

Figure S7. Molecular structures of other NFAs discussed in the manuscript: (a) ITIC, (b) 4TIC, (c) R10$4 \mathrm{Cl}$, (d) ITIC-2Cl- $\beta$, (e) $a$-ITIC-2Cl, (f) Y6 and (g) EH-IDTBR. 
Intermolecular short contacts between the LC conformers (Figure S8a) involve interactions between the nitrile carbon of the malononitrile group with the $\mathrm{C} 5$ hydrogen $(2.74 \AA)$ on the alkyl chain of a third LC ITIC-Th conformer. Similar to the intermolecular contacts between the LC ITIC-Th conformers, intermolecular contacts between the HC ITIC-Th conformers involve contacts between the IC units and hydrogens on the solubilizing chains of neighboring $\mathrm{HC}$ molecules. Within the same 2D layer, there are interactions between the nitrile carbon and C5 hydrogen ( $2.73 \AA$ ) (Figure S8b). Finally, in the case of two HC molecules at an angle to each other (Figure S8c) there are short intermolecular contacts (2.88 $)$ between the thiophene sulfur of the solubilizing chain of one molecule and the C5 hydrogen of the alkyl chain of another.
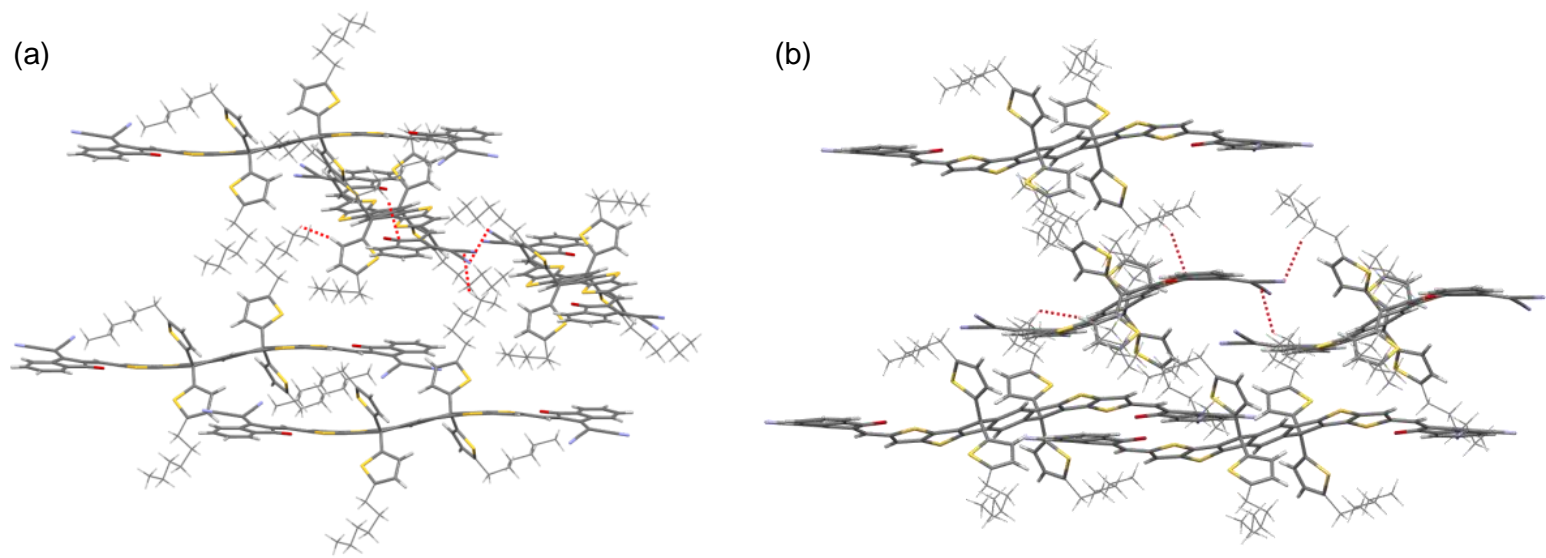

Figure S8. Intermolecular contacts between LC ITIC-Th conformers.

(a)

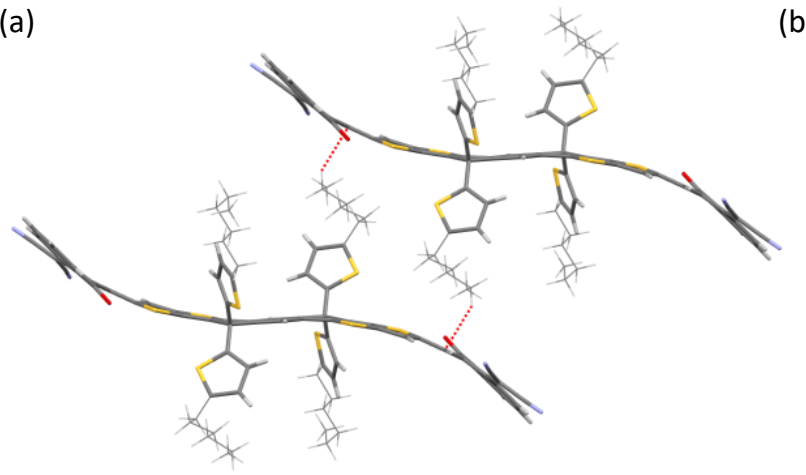

(b)

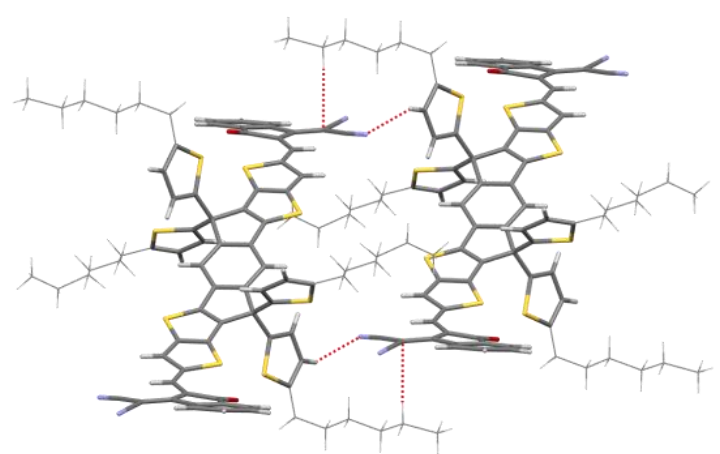

(c)

Figure S9. Intermolecular contacts between HC ITIC-Th conformers. 
The nitrogen of the LC ITIC-Th forms a short intermolecular contact $(2.64 \AA)$ with the 7-position hydrogen of the IC phenyl ring, as well as the carbonyl oxygen forming short intermolecular contacts with 4-position hydrogen of the IC phenyl ring of a second HC molecule (2.48 $\AA$ ). Furthermore, the 4-position hydrogen of the LC conformer forms a short intermolecular contact ( $2.29 \AA$ ) with the carbonyl oxygen of the other HC conformer. Finally, there are also short intermolecular contacts $(3.45 \AA)$ between the 6 position carbon of the LC conformer's IC phenyl ring and a sulfur of one of the HC conformer's pendant thiophene rings.

Intermolecular contacts between the overlapping LC and HC ITIC-Th conformers (Figure S10b) also feature a large amount of interactions involving sulfurs from the various thiophene rings present on both molecules, including instances of $S \cdots S$ interactions. The sulfur of the inner thiophene ring on the IDTT core of the LC conformer forms a short intermolecular contact $(2.60 \AA)$ with the hydrogen of a pendant thiophene, as well as $\mathrm{S} \cdots \mathrm{S}$ interaction (3.47 $\AA$ ) with the identical sulfur of the overlapping HC conformer.

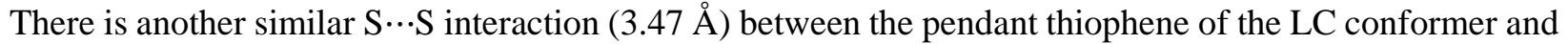
the outer thiophene ring of the IDTT core of the HC conformer, as well as two additional S $\cdots \mathrm{H}$ short contacts $(2.70 \AA, 2.74 \AA)$ between a thiophene ring and a hydrogen of a pendant thiophene. There are also short intermolecular contacts $(2.76 \AA, 2.67 \AA, 2.77 \AA)$ between the IC ring of the HC conformer and a hydrogen on the $\mathrm{C} 1$ carbon of the LC conformer's alkyl chain.

The HC and LC conformers are arranged diagonally relative to each other, with two general types of molecular contacts: end-to-end (Figure S10a) and overlapping (Figure S10b). In the case of end-to-end contacts between the LC and HC conformers, there are a number of short intermolecular contacts. The nitrogen of the LC ITIC-Th forms a short intermolecular contact (2.64 $\AA$ ) with the 7-position hydrogen of the IC phenyl ring, as well as the carbonyl oxygen forming short intermolecular contacts with 4-position hydrogen of the IC phenyl ring of a second HC molecule $(2.48 \AA)$. Furthermore, the 4-position hydrogen of the LC conformer forms a short intermolecular contact $(2.29 \AA)$ with the carbonyl oxygen of the other HC conformer. There are also short intermolecular contacts $(2.76 \AA$, $2.67 \AA, 2.77 \AA$ ) between the IC ring of the HC conformer and a hydrogen on the $\mathrm{C} 1$ carbon of the LC conformer's alkyl chain. Finally, there are short $S \cdots \mathrm{N}$ intermolecular contacts $(3.13 \AA$ ) between the same nitrogen and the sulfur of the inner thiophene ring of the HC conformer's IDTT core.

Intermolecular contacts between the overlapping LC and HC ITIC-Th conformers (Figure S10b) also feature a large amount of interactions involving sulfurs from the various thiophene rings present on both molecules, including instances of $S \cdots S$ interactions. The sulfur of the inner thiophene ring on the IDTT core of the LC conformer forms a short intermolecular contact $(2.60 \AA)$ with the hydrogen of a pendant thiophene, as well as $\mathrm{S} \cdots \mathrm{S}$ interaction $(3.47 \AA$ ) with the identical sulfur of the overlapping HC conformer.

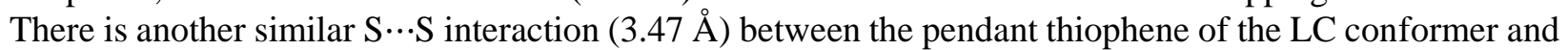
the outer thiophene ring of the IDTT core of the HC conformer, as well as two additional S $\cdots \mathrm{H}$ short contacts $(2.70 \AA, 2.74 \AA)$ between a thiophene ring and a hydrogen of a pendant thiophene.

Finally, in the case of two HC molecules at an angle to each other (Figure S9c) there are short intermolecular contacts $(2.88 \AA)$ between the thiophene sulfur of the solubilizing chain of one molecule and the C5 hydrogen of the alkyl chain of another.

Finally, there are short $\mathrm{S} \cdots \mathrm{N}$ intermolecular contacts $(3.13 \AA)$ between the same nitrogen and the sulfur of the inner thiophene ring of the HC conformer's IDTT core. 
(a)

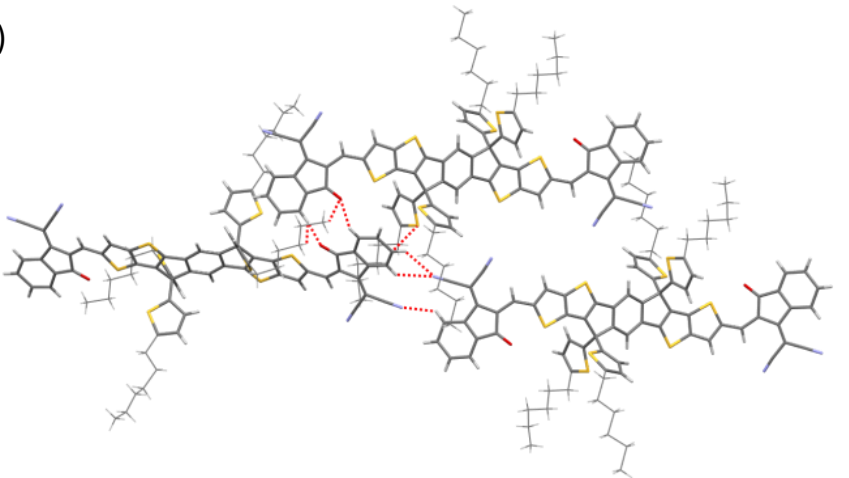

(b)

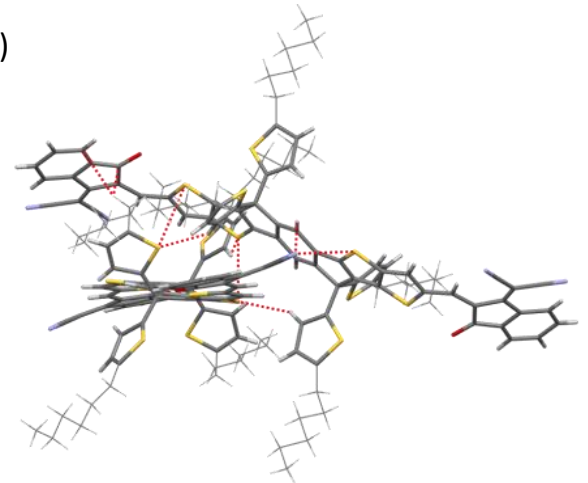

Figure S10. Intermolecular contacts between LC and HC ITIC-Th molecules: end-to-end (a) and overlapping (b). 


\section{S3 Hirshfeld surface analysis}

The fingerprint plot is a binned plot of distances between an atom internal to the Hirshfeld surface $\left(d_{i}\right)$ and an atom external to the surface $\left(d_{e}\right)$. Lighter colors represent more occurrences of contacts at a particular $\left(d_{i}, d_{e}\right)$ distance.

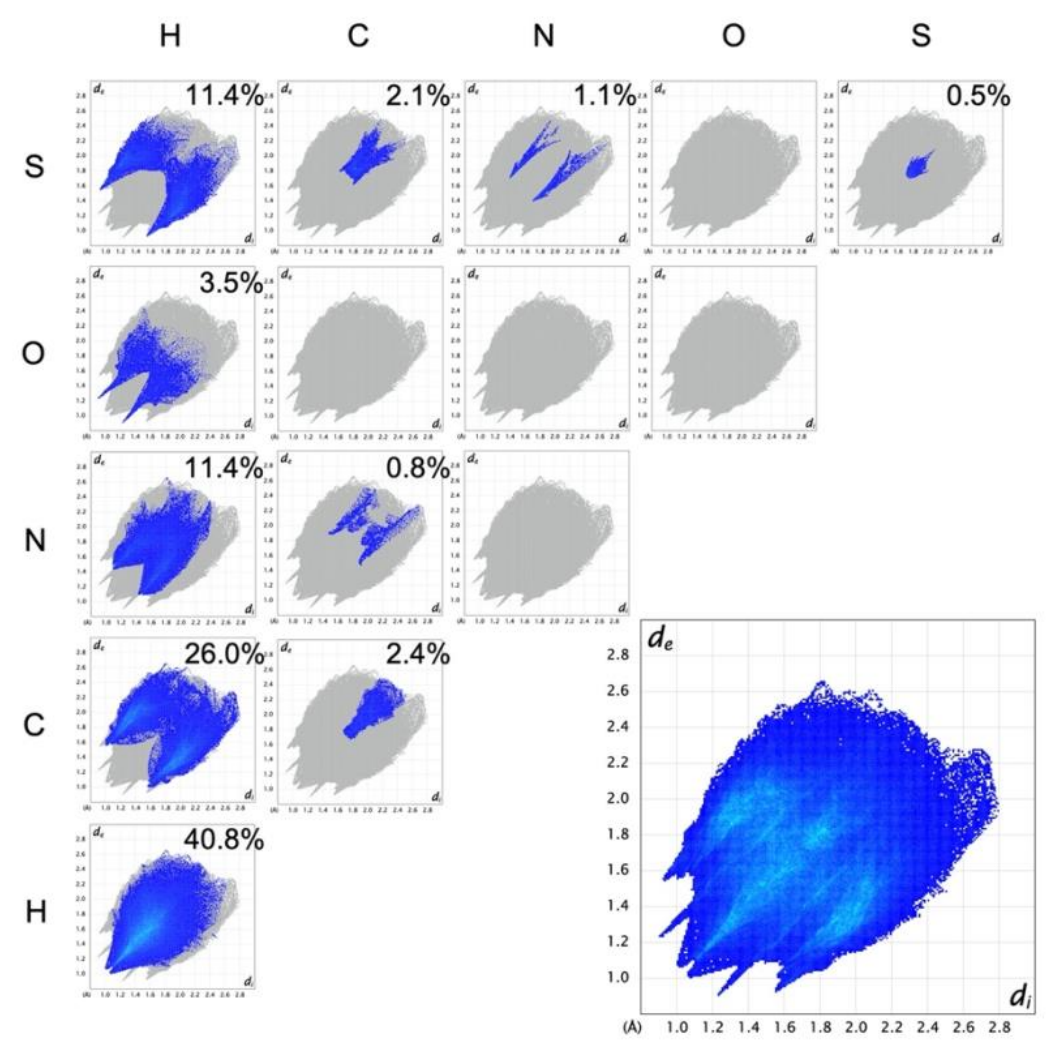

Figure S11. Hirshfeld surface fingerprint plot of the $\beta$-ITIC-Th polymorph, as well as decompositions of the fingerprint plot by specific atom-atom contacts. For each specific interaction, its contribution to the area of the Hirshfeld surface is given, except where it is $0 \%$. 


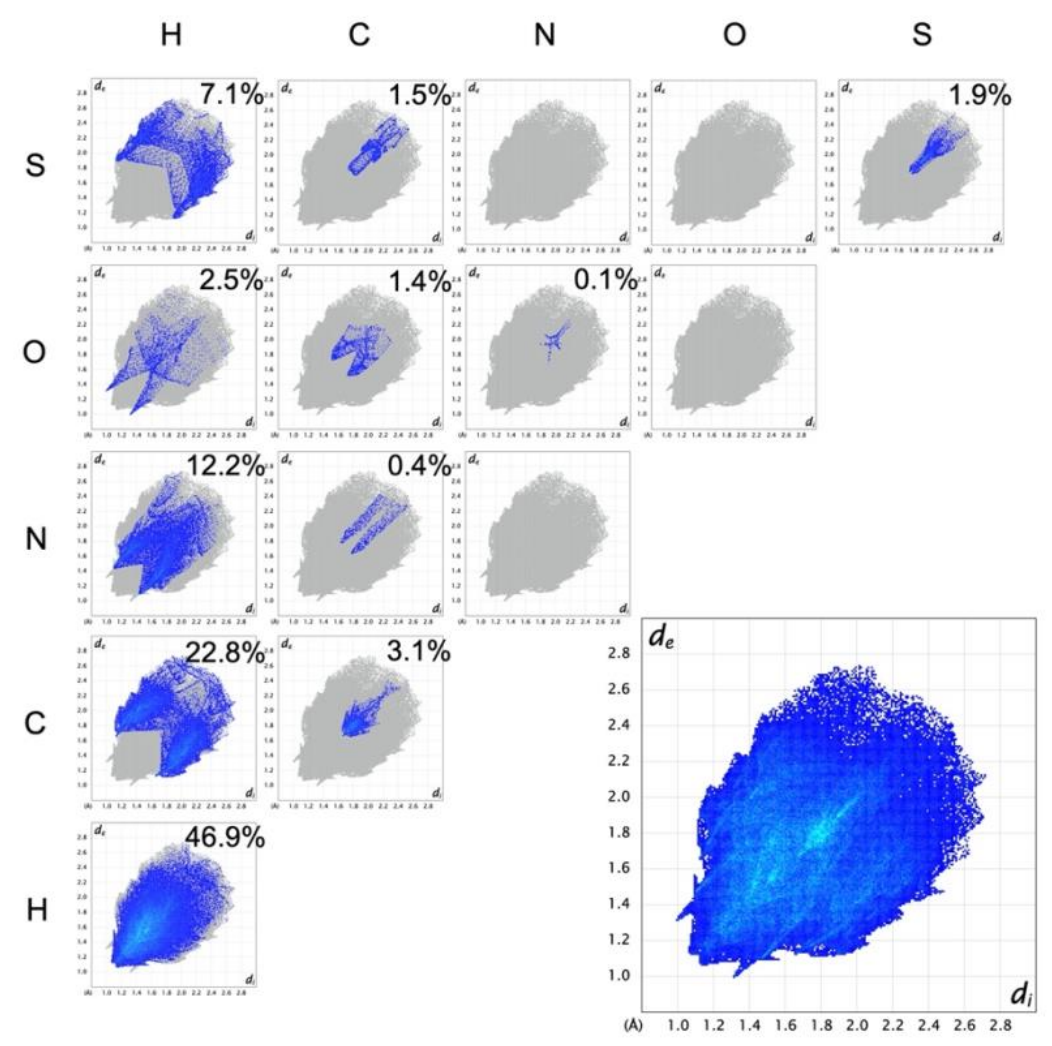

Figure S12. Hirshfeld surface fingerprint plot of the $\alpha$-ITIC-Th polymorph, as well as decompositions of the fingerprint plot by specific atom-atom contacts. For each specific interaction, its contribution to the area of the Hirshfeld surface is given, except where it is $0 \%$. 


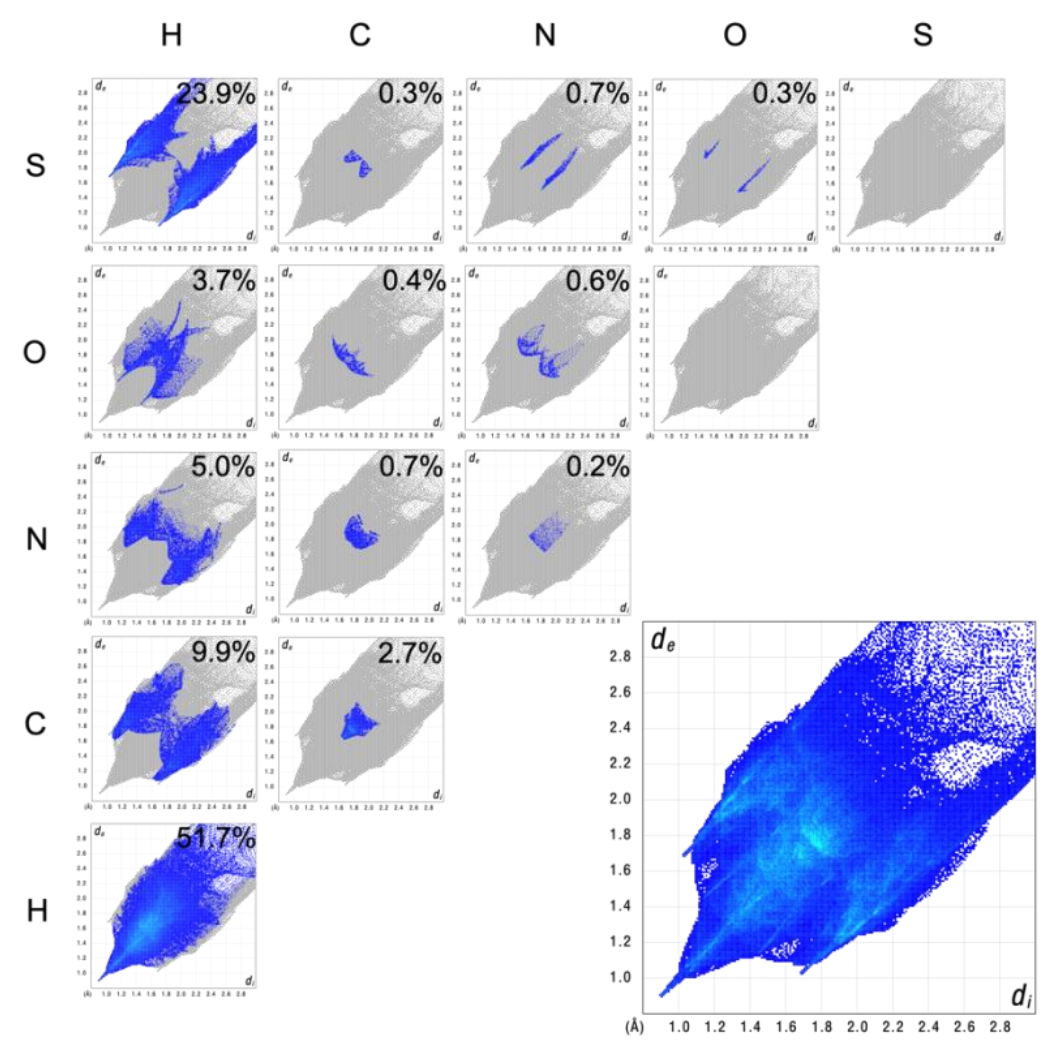

Figure S13. Hirshfeld surface fingerprint plot of EH-IDTBR, as well as decompositions of the fingerprint plot by specific atom-atom contacts. For each specific interaction, its contribution to the area of the Hirshfeld surface is given, except where it is $0 \%$. 


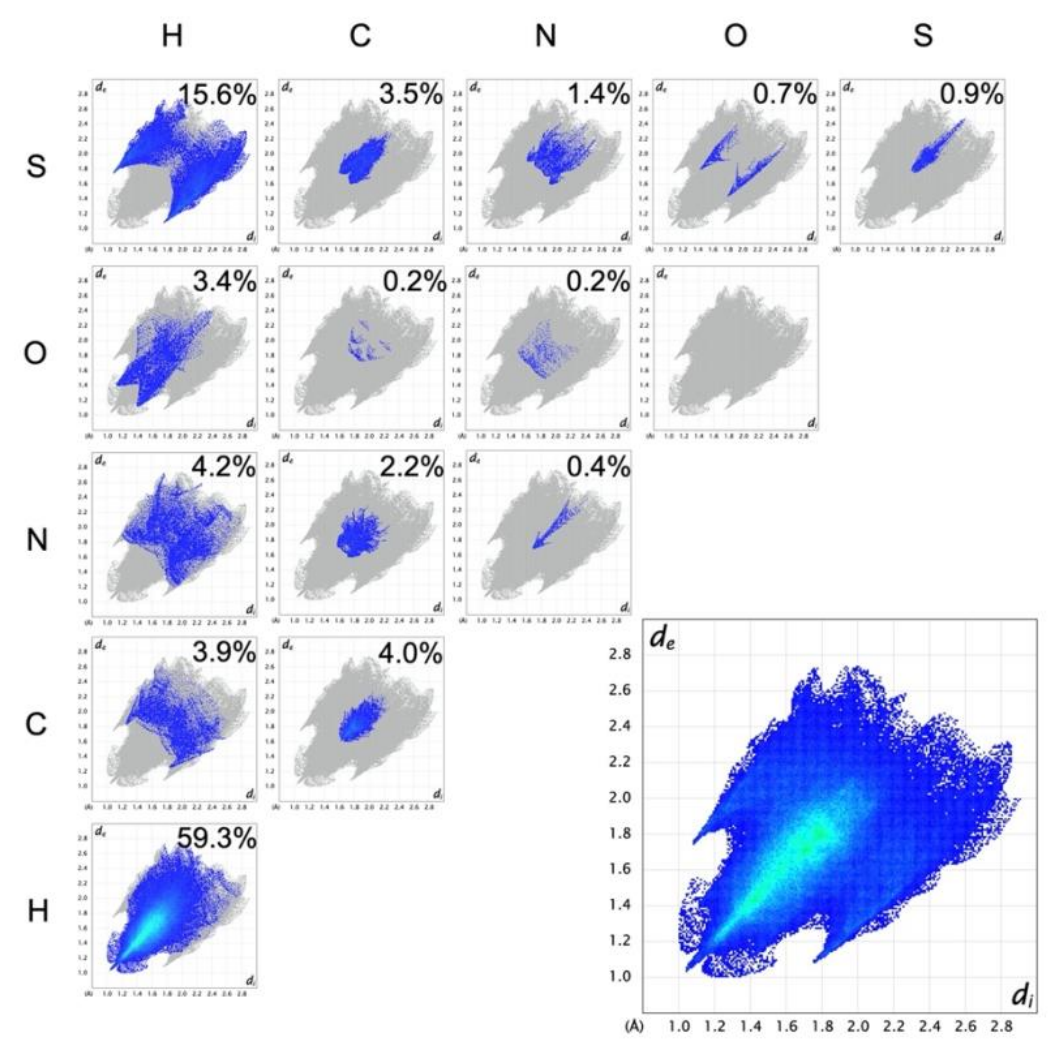

Figure S14. Hirshfeld surface fingerprint plot of the MicroED o-IDTBR atomic structure, as well as decompositions of the fingerprint plot by specific atom-atom contacts. For each specific interaction, its contribution to the area of the Hirshfeld surface is given, except where it is $0 \%$. 


\section{S4 Computational methodology}

\section{DFT calculations}

All density functional theory (DFT) calculations are performed in Gaussian $16^{1}$ and VASP $5.4^{2}$ packages. For molecules and their clusters we use CAM-B3LYP/6-31G* method (with D3 empirical corrections ${ }^{3}$ whenever appropriate) thoroughly benchmarked in our recent works. ${ }^{4-6}$ For crystals we mainly use the PBE functional ${ }^{7}$ with $\mathrm{D} 3$ empirical corrections ${ }^{3}$ and projector-augmented wave (PAW) pseudopotentials ${ }^{8}$ with at least $400 \mathrm{eV}$ plane-wave cutoff (abbreviated as PBE-D3/PAW400). More specifically, we use a $400 \mathrm{eV}$ cutoff for fixed cell relaxation, $600 \mathrm{eV}$ for fixed volume relaxation, and $900 \mathrm{eV}$ for unit cell relaxation. For most of the structures, the DFT-optimized geometry is obtained in two steps: first, we optimize the unit cell (and atomic positions) using $900 \mathrm{eV}$ cutoff, and then reoptimize atomic positions using $400 \mathrm{eV}$ cutoff, because accurate calculation of total energies is computationally prohibitive at $900 \mathrm{eV}$ cutoff, whereas unit cells optimized at lower cutoffs are inaccurate. For a subset of crystals we perform robust unit cell optimization using $4^{\text {th }}$ order polynomial fit of energy for a set of fixed-volume relaxations with $600 \mathrm{eV}$ cutoff, see Figure S15. Only in one case this method showed a preference over simple relaxation with $900 \mathrm{eV}$ cutoff, because it allowed to go out of shallow minima in potential energy landscape. All geometry optimizations are performed with tight criteria predefined in both Gaussian and VASP programs. Final gradients are listed in Table S2. A $\Gamma$-centered Monkhorst-Pack grid with k-spacing not exceeding $0.2 \AA^{-1}$ (e.g. $2 \times 2 \times 1$ for o-IDTBR) and Gaussian smearing is used during geometry relaxation. Single point energy is calculated with doubled grid (e.g. $4 \times 4 \times 2$ for oIDTBR) and tetrahedron smearing method with Blochl corrections. As additional benchmarking we redo some calculations with vdW-DF2 functional. ${ }^{9,10}$

\section{Coarse graining of electronic Hamiltonian}

The coarse-grained Hamiltonian for holes is calculated using well established methodology. ${ }^{15}$ In case of electrons, a minimal reasonable model includes two sites per molecule. Therefore we use a more sophisticated approach introduced in ref. $\left[{ }^{16}\right]$, partitioning the o-IDTBR backbone into the central electron-donor thienoacene block and the two terminal electron-acceptor blocks which contain $80 \%$ of the electronic density of the four lowest LUMOs. It should be noted that for the experimental geometry the onsite energies of the two sites differ by $0.3 \mathrm{eV}$, whereas for the PBE-D3 relaxed geometries (both fixed cell and optimized cell) the sites are nearly identical (less than $0.5 \mathrm{meV}$ difference). For this reason, in calculations we assume that all sites have the same energy. Signs of inter-site electronic couplings are fixed by the sign of the polaron wave-function in Figure S16, so that if $\phi$ is the molecular orbital localized as in Figure S16 then, e.g., the LUMO of the molecule is $\phi_{1}+\phi_{2}$ where indices denote the two opposite ends of the molecule.

\section{Estimation of charge carrier mobility}

In the hopping regime the zero-field charge carrier mobility relates to the diffusion tensor by the Einstein relation: ${ }^{15}$

$$
\mu=\frac{e}{T} D
$$

where $T$ is the temperature. Let $D(w)$ be diffusion tensor as function of rates $w$ between all pairs of sites. In the second order perturbation theory over inter-site electronic couplings $t$, the rates are given by

$$
w=\frac{2 \pi}{\hbar} t^{2} J
$$

where $J$ is the spectral overlap (convolution of the spectral densities of the initial and final electronic states). ${ }^{15}$ Then

$$
\mu=\frac{2 \pi \mathrm{e}}{\hbar} \times D\left(t^{2}\right) \times \frac{1}{W(T)^{2}}
$$


where $D\left(t^{2}\right)$ is the hopping amplitude tensor obtained by formal replacement of rates $w$ by $t^{2}$ in $D(w)$ function, and $W(T)=\sqrt{T / J(T)}$. In the case where $J$ and $t$ are disordered or fluctuate, a proper average should be used in Eq. (S2). Square roots of eigenvalues of $D\left(t^{2}\right)$ are the three hopping amplitudes. Note that for the simple cubic lattice

$$
D\left(t^{2}\right)=(a \cdot t)^{2}
$$

where $a$ is the hopping distance, that is why the product $\eta=a \cdot t$ is called the hopping amplitude. If distances $a$ are measured in Angstroms then

$$
\mu=0.9546 \ldots \frac{D\left(t^{2}\right)}{W(T)^{2}} \frac{\mathrm{cm}^{2}}{\mathrm{~V} \cdot \mathrm{s}}
$$

Importantly, in the simplest approximation the numerator $D$ depends only on the electronic structure (and geometry), whereas $W$ depends only on electron-phonon couplings (and temperature).

In the regime of fully delocalized charge carriers the mobility can be approximated by the Drude formula

$$
\mu=\frac{e \tau_{\text {scattering }}}{m_{\text {effective }}}
$$

where $m_{\text {effective }}$ depends only on electronic structure and $\tau_{\text {scattering }}$ accounts for electron-phonon coupling. In this regime the electronic bandwidth (mean square root of electronic level variations) and bandspan (maximum minus minimum energy of band electrons) are useful simple descriptors of charge carrier transport. 


\section{S5 Calculated structural properties}
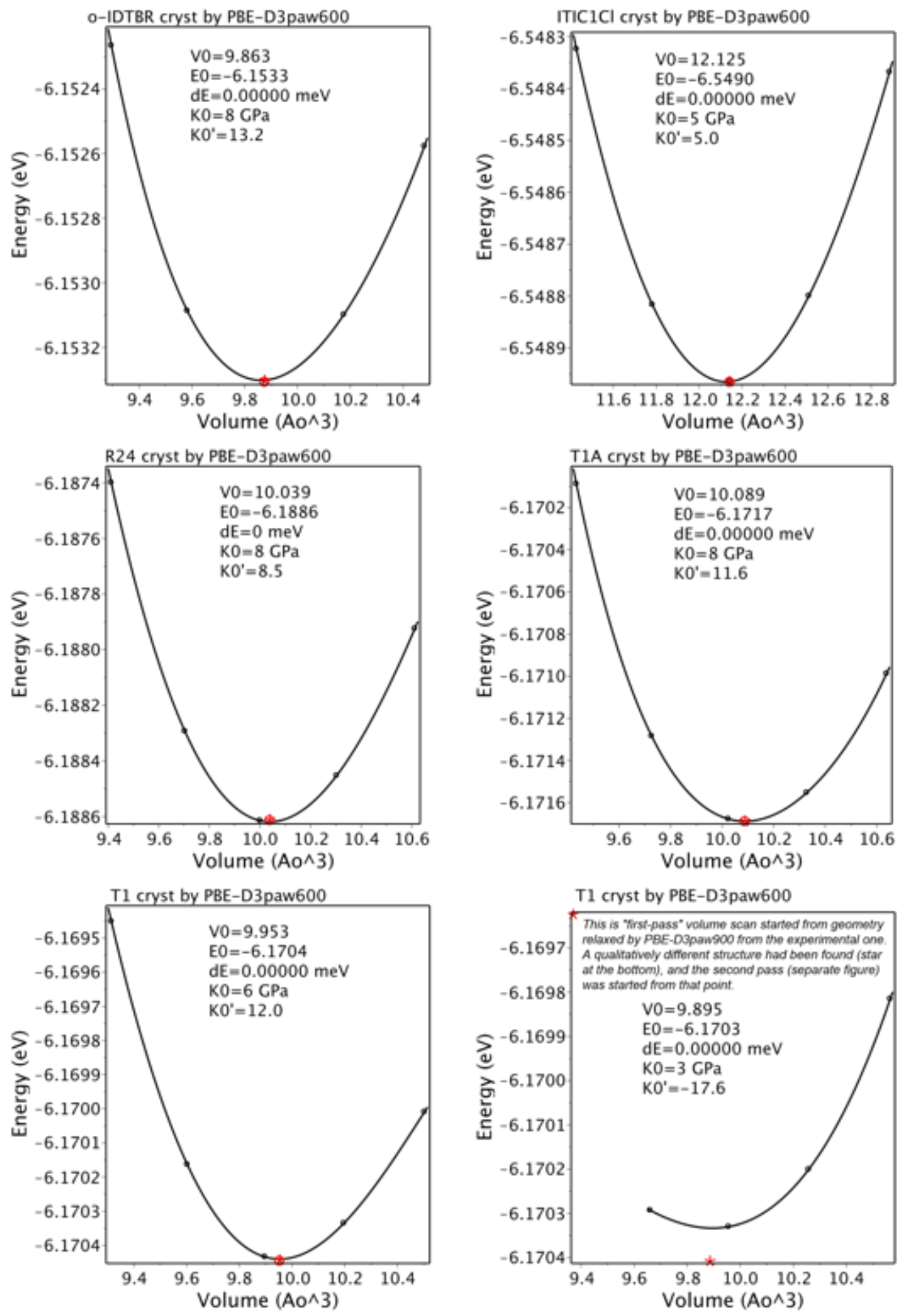

Figure S15. Volume scans fitted by $4^{\text {th }}$ order polynomial. Main parameters of the equation of state are shown including bulk modulus $\mathrm{K}_{0}$ and its pressure-derivative $\mathrm{K}_{0}{ }^{\prime}$ at equilibrium. 
Table S2. Comparison of experimental geometry and geometry optimized by PBE-D3/PAW400 at fixed cell. Listed are number of molecules per unit cell, number of atoms per molecule, space group and deviations of the geometry of conjugated backbone: root mean square (RMS) deviation of superimposed Cartesian coordinates $\left(C_{R M S}\right)$, RMS of bond lengths $\left(B_{R M S}\right)$, minimum and maximum deviation of bond lengths $\left(B_{\min }\right.$ and $\left.B_{\max }\right)$. For crystals with two symmetry-nonequivalent molecules, the two molecules are compared separately. Also final gradient $\left(G_{R M S}\right.$ and $\left.G_{\max }\right)$ and binding energy ( $\left.E_{\text {binding }}\right)$ are given. The latter is calculated with respect to the energy of the molecule in vacuum fully relaxed from its geometry in the crystal. Four relevant published crystals are given for reference.

\begin{tabular}{|c|c|c|c|c|c|c|c|c|c|c|}
\hline Crystal & $\begin{array}{l}\mathrm{mol} \\
/ \mathrm{UC}\end{array}$ & $\begin{array}{c}\text { atoms } \\
/ \mathrm{mol}\end{array}$ & $\begin{array}{l}\text { space } \\
\text { group }\end{array}$ & $\begin{array}{c}C_{R M S} \\
\AA\end{array}$ & $\begin{array}{c}B_{R M S} \\
\AA\end{array}$ & $\begin{array}{c}B_{\min } \\
\AA\end{array}$ & $\begin{array}{c}B_{\max } \\
\AA\end{array}$ & $\begin{array}{c}G_{R M S} \\
\mathrm{meV} / \AA\end{array}$ & $\begin{array}{c}G_{\max } \\
\mathrm{meV} / \AA\end{array}$ & $\begin{array}{l}E_{\text {binding }} \\
\mathrm{eV} / \mathrm{mol}\end{array}$ \\
\hline o-IDTBR & 4 & 176 & $\mathrm{P} 21 / \mathrm{c}$ & .049 & .038 & -.08 & .11 & 2.3 & 14 & 4.83 \\
\hline \multirow[t]{2}{*}{ ITIC-Th } & 4 & 174 & $\mathrm{P} 21 / \mathrm{c}$ & .111 & .042 & -.03 & .10 & 2.5 & 21 & 5.01 \\
\hline & & & & .120 & .045 & -.05 & .11 & & & \\
\hline$p$-DTS $\left(\mathrm{PTTH}_{2}\right)_{2}\left[{ }^{11}\right]$ & 4 & 149 & $\mathrm{C} 2 / \mathrm{c}$ & .068 & .018 & -.02 & .05 & 2.3 & 8 & 4.53 \\
\hline$p$-DTS(FBTTH $\left.{ }_{2}\right)_{2}\left[{ }^{12,13}\right]$ & 2 & 151 & P-1 & .123 & .048 & -.09 & .11 & 2.6 & 14 & 4.18 \\
\hline \multirow[t]{2}{*}{$a$-ITIC-2Cl $\left[{ }^{14}\right]$} & 4 & 186 & $\mathrm{P}-1$ & .110 & .025 & -.04 & .07 & 2.5 & 21 & 4.74 \\
\hline & & & & .346 & .025 & -.04 & .07 & & & \\
\hline \multirow[t]{2}{*}{ ITIC-2Cl- $\beta\left[{ }^{14}\right]$} & 2 & 186 & P-1 & .052 & .018 & -.02 & .04 & 3.8 & 31 & 4.61 \\
\hline & & & & .055 & .018 & -.03 & .04 & & & \\
\hline
\end{tabular}


Table S3. Comparison of experimental geometry and geometry fully relaxed by DFT-D. Listed are volume per atom, unit cell parameters and binding energy. The relative binding energy is calculated with respect to the average energy of molecular conformers observed in the crystal but optimized in vacuum, allowing to obtain intermolecular packing energy. For the absolute binding energy a single conformer is used (typically with unfolded side chains, except for EH-IDTBR where such conformer is ill-defined), thus allowing to compare crystalline polymorphs. For PAW400 geometries the unit cell is obtained by full geometry relaxation at $900 \mathrm{eV}$ cutoff, whereas for PAW600 geometries volume scan is used.

\begin{tabular}{|c|c|c|c|c|c|c|c|c|c|}
\hline Crystal & $\begin{array}{c}\mathrm{V}_{1} \\
\left(\AA^{3} / \text { atom }\right)\end{array}$ & a $(\AA)$ & $\mathrm{b}(\AA)$ & c $(\AA)$ & $\alpha\left(^{\circ}\right)$ & $\beta\left(^{\circ}\right)$ & $\gamma\left(\left(^{\circ}\right)\right.$ & $\begin{array}{c}E_{\text {binding }} \text { relative } \\
(\mathrm{eV} / \mathrm{mol})\end{array}$ & $\begin{array}{c}E_{\text {binding }}{ }^{\text {absolute }} \\
(\mathrm{eV} / \mathrm{mol})\end{array}$ \\
\hline \multicolumn{10}{|c|}{ o-IDTBR } \\
\hline Experiment & 9.95 & 13.73 & 15.68 & 32.72 & 90 & 95.9 & 90 & & \\
\hline PBE-D3/PAW400 & 9.86 & 13.70 & 15.58 & 32.65 & 90 & 95.2 & 90 & 4.84 & 4.84 \\
\hline PBE-D3/PAW600 & 9.88 & 13.72 & 15.58 & 32.67 & 90 & 95.3 & 90 & 4.82 & 4.82 \\
\hline \multicolumn{10}{|c|}{ o-IDTBR (X-ray) } \\
\hline Experiment & 10.05 & 13.77 & 15.81 & 16.37 & 90 & 96.3 & 90 & & \\
\hline PBE-D3/PAW400 & 9.97 & 13.80 & 15.63 & 32.71 & 90 & 96.0 & 90 & 4.80 & 4.80 \\
\hline \multicolumn{10}{|c|}{ EH-IDTBR } \\
\hline Experiment & 10.09 & 10.07 & 12.37 & 16.37 & 78.5 & 73.1 & 66.1 & & \\
\hline PBE-D3/PAW400 & 10.00 & 9.64 & 12.55 & 16.42 & 79.3 & 73.2 & 68.4 & 4.05 & \\
\hline \multicolumn{10}{|c|}{$\beta$-ITIC-Th } \\
\hline Experiment & 9.78 & 15.32 & 29.00 & 15.46 & 90 & 97.5 & 90 & & \\
\hline PBE-D3/PAW400 & 10.23 & 15.55 & 29.50 & 15.64 & 90 & 96.9 & 90 & 5.32 & 5.38 \\
\hline \multicolumn{10}{|c|}{$\alpha$-ITIC-Th (X-ray) } \\
\hline Experiment & 10.36 & 8.92 & 12.48 & 16.90 & 84.4 & 83.1 & 74.5 & & \\
\hline PBE-D3/PAW400 & 10.28 & 8.92 & 12.40 & 16.95 & 84.6 & 83.3 & 74.3 & 5.38 & 5.70 \\
\hline \multicolumn{10}{|c|}{ ITIC } \\
\hline Experiment & 10.10 & 14.88 & 15.47 & 18.08 & 99.3 & 101.5 & 108.4 & & \\
\hline PBE-D3/PAW400 & 10.14 & 14.91 & 15.48 & 18.10 & 99.2 & 101.4 & 108.5 & 5.25 & 5.38 \\
\hline \multicolumn{10}{|c|}{$\beta$-ITIC-2Cl } \\
\hline Experiment & 12.17 & 8.07 & 23.77 & 24.21 & 80.2 & 87.0 & 81.6 & & \\
\hline PBE-D3/PAW400 & 12.14 & 8.02 & 23.82 & 24.26 & 80.2 & 88.0 & 81.7 & 4.63 & 4.63 \\
\hline PBE-D3/PAW600 & 12.14 & 8.02 & 23.82 & 24.26 & 80.2 & 88.0 & 81.7 & 4.61 & 4.61 \\
\hline \multicolumn{10}{|c|}{$\alpha$-ITIC-2Cl } \\
\hline Experiment & 11.63 & 14.96 & 21.06 & 28.35 & 102.8 & 96.4 & 90.4 & & \\
\hline PBE-D3/PAW400 & 11.30 & 14.92 & 20.66 & 28.17 & 102.9 & 96.6 & 89.9 & 4.87 & 4.86 \\
\hline
\end{tabular}




\section{S6 Calculated electronic properties}

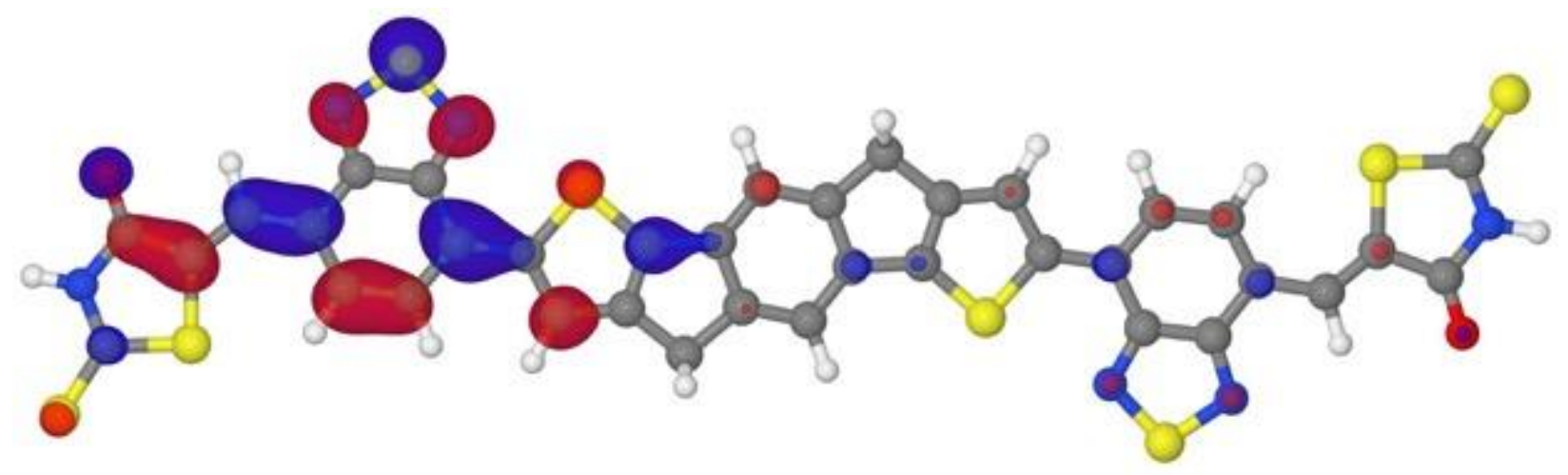

Figure S16. Electron polaron wave-function (natural orbital with occupation 1 of the relaxed anion). The asymmetric configuration is $12 \mathrm{meV}$ lower in energy than the symmetric one $(0.35 \mathrm{eV}$ in a solvent with high dielectric constant). 
Table S4. Multiplicities and transfer integrals of NFAs calculated at the experimental geometry. In the case where the difference between the optimized and experimental unit cells influences the transfer integral, the value is listed in curly braces.

\begin{tabular}{|c|c|c|c|}
\hline Monomer & Mul. & $t(\mathrm{meV})$ & \\
\hline \multicolumn{4}{|c|}{ o-IDTBR (MicroED) } \\
\hline Intramol. & 8 & 89 & \\
\hline $40-11$ & 4 & 110 & \\
\hline 2001 & 8 & 91 & \\
\hline 4100 & 4 & 25 & \\
\hline \multicolumn{4}{|c|}{ EH-IDTBR } \\
\hline Intramol. & 2 & 83 & \\
\hline 1011 & 2 & 102 & \\
\hline 1001 & 2 & 38 & \\
\hline \multicolumn{4}{|c|}{$\beta$-ITIC-2Cl } \\
\hline Intramol. & 2 & 109 & \\
\hline Intramol.' & 2 & 118 & \\
\hline 2000 & 4 & 41 & \\
\hline 2100 & 4 & 23 & \\
\hline \multicolumn{4}{|c|}{$\alpha$-ITIC-2Cl } \\
\hline Intramol. & 4 & 150 & \\
\hline Intramol.' & 4 & 150 & \\
\hline $4^{\prime}-111$ & 2 & 118 & $\{146\}$ \\
\hline $200-1$ & 4 & 161 & $\{143\}$ \\
\hline 2000 & 4 & 99 & $\{122\}$ \\
\hline $311-1$ & 2 & 141 & \\
\hline 4' 000 & 2 & 116 & \\
\hline 3000 & 2 & 84 & \\
\hline \multicolumn{4}{|c|}{$\beta$-ITIC-Th (MicroED) } \\
\hline $41-1-1$ & 8 & 4 & \\
\hline 2000 & 8 & 6 & \\
\hline \multicolumn{4}{|c|}{$\alpha$-ITIC-Th } \\
\hline Intramol. & 2 & 120 & \\
\hline $101-1$ & 2 & 71 & \\
\hline \multicolumn{4}{|c|}{ ITIC } \\
\hline 2000 & 4 & 19 & \\
\hline
\end{tabular}


Table S4. Comparison of tight-binding parameters (in $\mathrm{meV}$ ) for electrons derived from different clusters: from monomer to tetramer. Onsite energies for the left and right ends of the molecule, $\mathrm{L}_{\mathrm{L}}$ and $\mathrm{R}$, are counted from the reference energy $-2.34 \mathrm{eV}$. Multiple values per multimer are given for all symmetry inequivalent monomers. Asterisks $(*)$ mark contact sites. The tetramer $1+2+3+4$ is a part of the $\pi$-stack along $(\xi, 1 / 2,0)$ direction (the translational period is 4 molecules). The geometry is taken at fixed experimental cell but with relaxed atomic positions. The L-R asymmetry is negligible in the monomer $(0.2 \mathrm{meV})$ but is about $50 \mathrm{meV}$ in tetramers.

\begin{tabular}{|c|c|c|c|c|c|c|c|}
\hline cluster & contacts & $\mathrm{L}$ & $\mathrm{R}$ & $t_{\text {intramol }}$ & $t_{\mathrm{LL}}$ & $t_{\mathrm{LR}}$ & $t_{\mathrm{RR}}$ \\
\hline monomer & & +0.1 & -0.1 & 130 & - & - & - \\
\hline \multirow[t]{2}{*}{ dimer $1+2$} & LR & $-12^{*}$ & +36 & 130 & - & 115 & - \\
\hline & & +41 & $-32 *$ & 131 & & & \\
\hline dimer $2+3$ & $\mathrm{RR}$ & +14 & $-83 *$ & 129 & - & - & 123 \\
\hline dimer $4+1$ & LL & $0 *$ & +13 & 129 & 31 & - & - \\
\hline \multirow[t]{3}{*}{ trimer $1+2+3$} & LRR & $-43^{*}$ & +2 & 130 & - & 118 & - \\
\hline & & +51 & $-99 *$ & 131 & - & - & 121 \\
\hline & & -27 & $-112^{*}$ & 129 & & & \\
\hline \multirow[t]{3}{*}{ trimer $4+1+2$} & LLR & $-58^{*}$ & -13 & 129 & 32 & - & - \\
\hline & & $-21^{*}$ & +45 & 129 & - & 110 & - \\
\hline & & +8 & $-38 *$ & 131 & & & \\
\hline \multirow[t]{4}{*}{ tetramer $4+1+2+3$} & LLRR & $-57^{*}$ & -2 & 129 & 32 & - & - \\
\hline & & $-50^{*}$ & +12 & 129 & - & 113 & - \\
\hline & & +17 & $-104 *$ & 130 & - & - & 121 \\
\hline & & -20 & $-119 *$ & 129 & & & \\
\hline \multirow[t]{2}{*}{ tetramer $1+2+3+4$} & LRRL & $-68 *$ & +1 & 130 & - & 118 & - \\
\hline & & +11 & $-122 *$ & 130 & - & - & 119 \\
\hline \multirow[t]{2}{*}{ tetramer $3+4+1+2$} & RLLR & +5 & $-75^{*}$ & 131 & - & 112 & - \\
\hline & & $-71^{*}$ & +20 & 129 & 34 & - & - \\
\hline
\end{tabular}




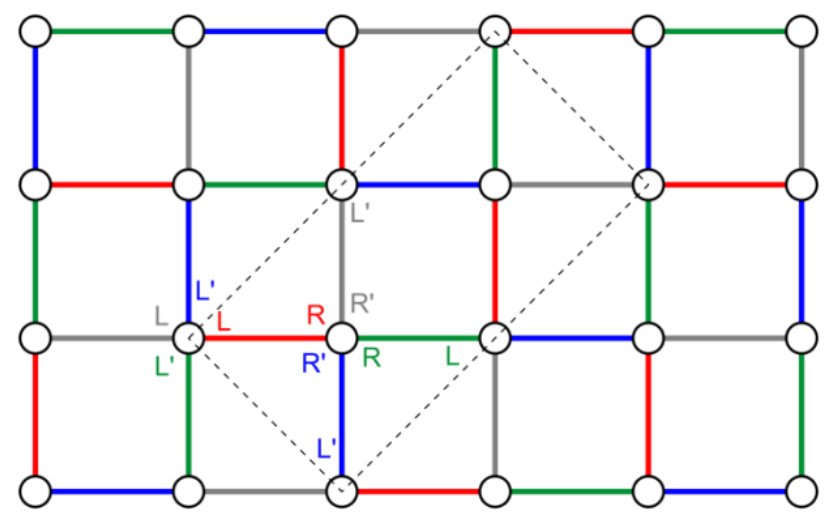

Figure S17. Schematic representation of the $a$-ITIC-2Cl wire mesh topology along with representation of the different types of contacts. There are two symmetry inequivalent molecules in the unit cell, distinguished by a prime. The unit cell is represented by the dashed lines. 
(a)

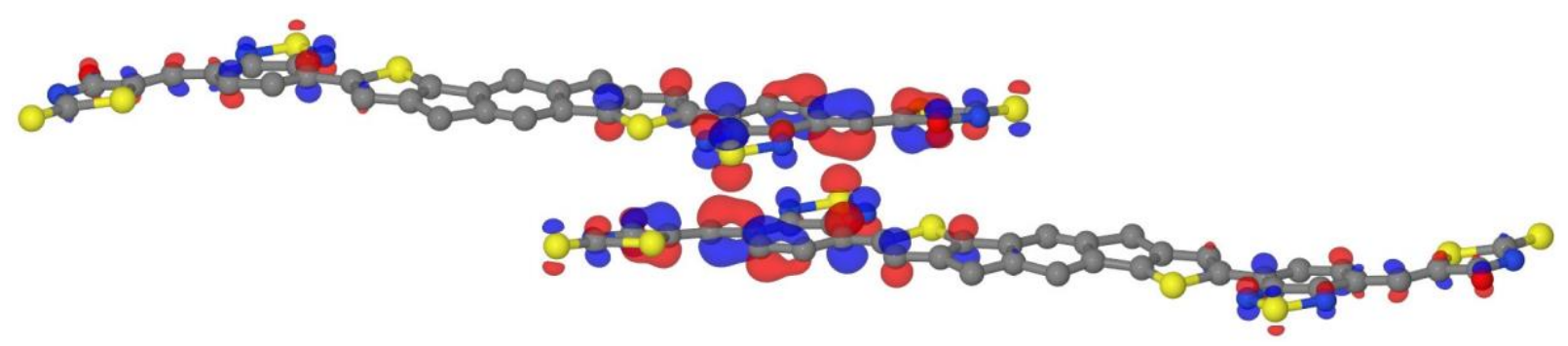

(b)

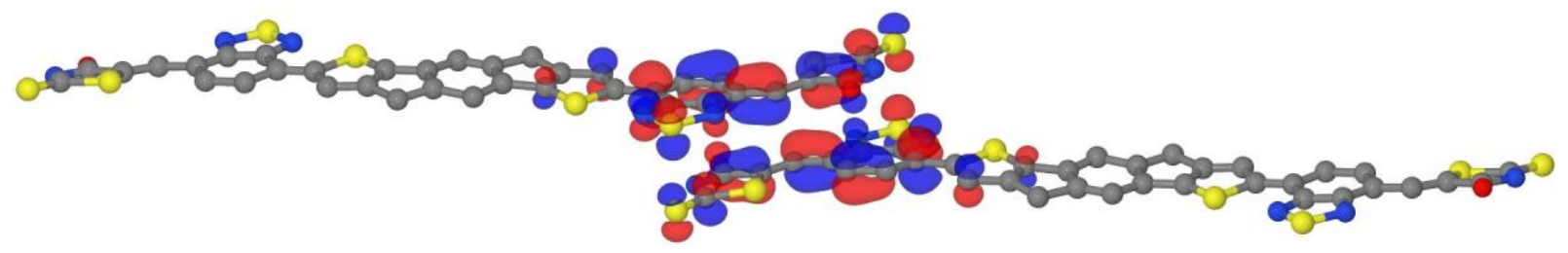

(c)

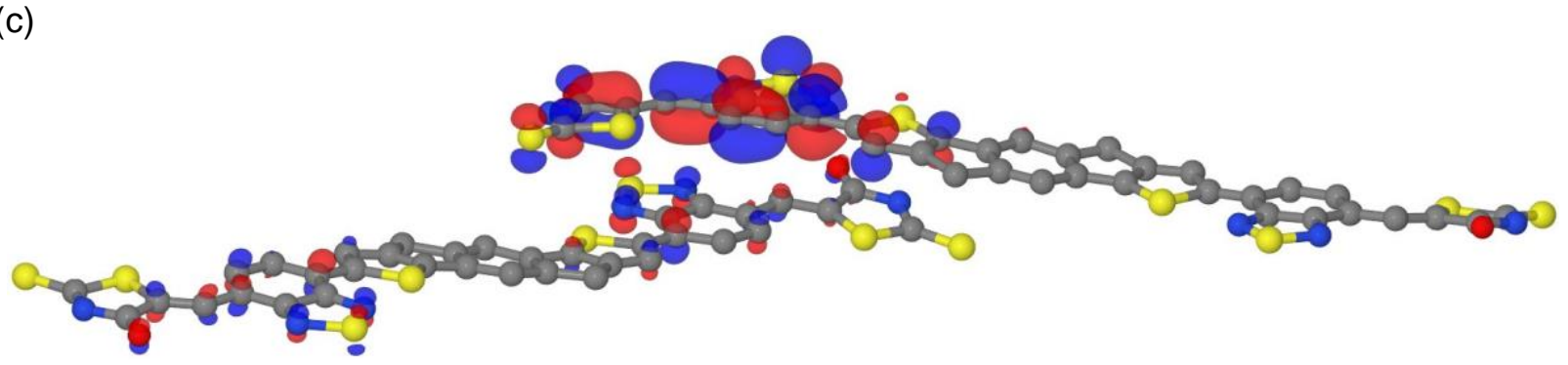

Figure S18. DFT-calculated LUMO distributions for the (a) RR, (b) LL and (c) LR = RL pairs in the oIDTBR MicroED structure. 
(a)

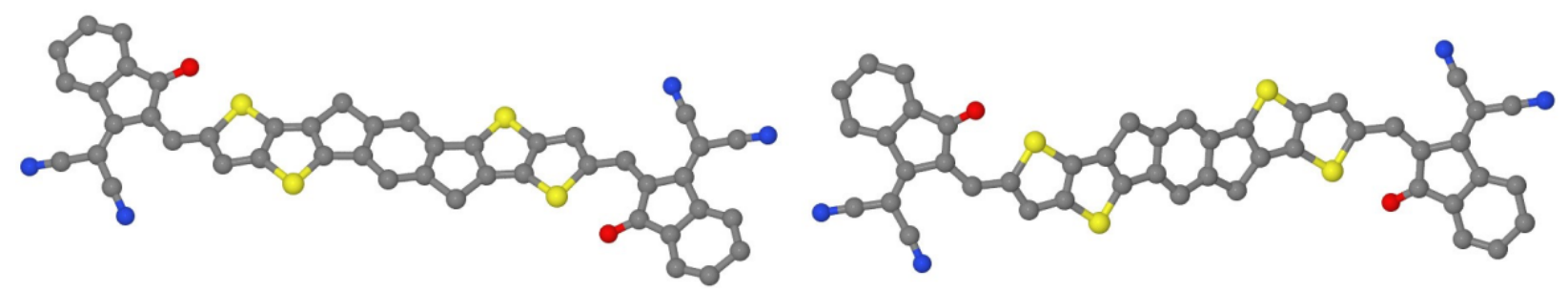

(b)

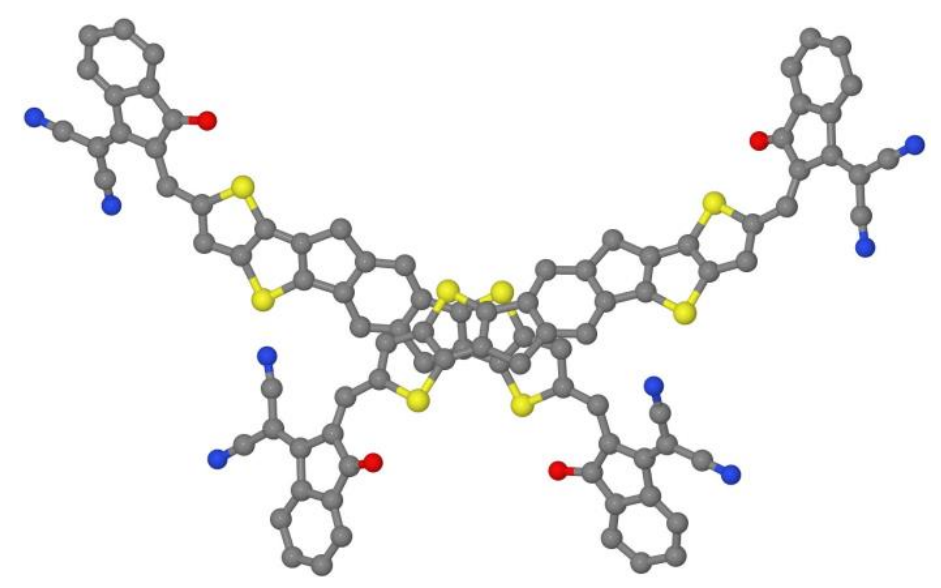

Figure S19. The (a) $1000+2000$ and (b) $1000+41-1-1$ pairs of ITIC-Th molecules in the MicroEDdetermined structure. 
Table S6. Electronic descriptors of charge carrier transport: o-IDTBR compared to other systems studied by the same methodology. Use of experimental geometries gives consistent values of the descriptors for all considered here systems (o-IDTBR is shown as example). The second column "cc" indicates the charge carrier: ' $\mathrm{e}$ ' for electron and ' $\mathrm{h}$ ' for hole. The three hopping amplitudes $\eta_{1,2,3}$ are ordered by value. The geometry type "H-poor" include molecules with small number of terminal hydrogens and thus having large intermolecular electronic couplings in non- $\pi$-stacking directions often resulting in good 3D connectivity. The "model" systems correspond to o-IDTBR and Y6 crystals with absolute values of all inter-site electronic couplings set to $100 \mathrm{meV}$.

\begin{tabular}{|c|c|c|c|c|c|c|c|c|}
\hline system & $\mathrm{cc}$ & $\begin{array}{c}\eta_{1} \\
(\mathrm{eV} \cdot \AA)\end{array}$ & $\begin{array}{c}\eta_{2} \\
(\mathrm{eV} \cdot \AA)\end{array}$ & $\begin{array}{c}\eta_{3} \\
(\mathrm{eV} \cdot \AA)\end{array}$ & $\begin{array}{c}\text { bandspan } \\
(\mathrm{eV})\end{array}$ & $\begin{array}{c}\text { bandwidth } \\
(\mathrm{eV})\end{array}$ & geometry & connectivity \\
\hline \multicolumn{9}{|c|}{ 1D systems } \\
\hline $\mathrm{R} 24$ & $\mathrm{~h}$ & 0.33 & 0.06 & 0.03 & 0.26 & 0.09 & $\pi$-stack & $1 \mathrm{D}$ \\
\hline $\mathrm{T} 1$ & $\mathrm{~h}$ & 1.23 & 0.01 & 0.00 & 0.54 & 0.19 & slipped stack & $1 \mathrm{D}$ \\
\hline EH-IDTBR & $\mathrm{e}$ & 1.32 & 0.22 & 0.03 & 0.55 & 0.17 & slipped stack & $1 \mathrm{D}$ \\
\hline ITIC-Th & $\mathrm{e}$ & 0.94 & 0.08 & 0.00 & 0.53 & 0.19 & slipped stack & $1 \mathrm{D}$ \\
\hline ITIC- $1 \mathrm{Cl}$ & $\mathrm{e}$ & 0.74 & 0.07 & 0.00 & 0.46 & 0.17 & broken mesh & $1 \mathrm{D}$ \\
\hline \multicolumn{9}{|c|}{ 2D systems } \\
\hline hexacene & $\mathrm{h}$ & 0.75 & 0.65 & 0.04 & 1.08 & 0.28 & herringbone & triangular \\
\hline TIPS-pentacene & $\mathrm{e}$ & 1.39 & 0.53 & 0.00 & 0.98 & 0.26 & brickwork & oblique \\
\hline \multicolumn{9}{|c|}{ 3D systems } \\
\hline F2-TCNQ & $\mathrm{e}$ & 0.94 & 0.39 & 0.38 & 1.02 & 0.18 & H-poor & $3 \mathrm{D}$ \\
\hline o-IDTBR (exp.geom.) & $\mathrm{e}$ & 0.77 & 0.29 & 0.16 & 0.51 & 0.15 & "wire mesh" & Fig. S4 \\
\hline o-IDTBR & $\mathrm{e}$ & 1.04 & 0.35 & 0.19 & 0.65 & 0.19 & & \\
\hline o-IDTBR model & $\mathrm{e}$ & 0.99 & 0.39 & 0.33 & 0.60 & 0.17 & & \\
\hline Y6 model & $\mathrm{e}$ & 0.72 & 0.61 & 0.31 & 0.60 & 0.17 & & \\
\hline Y6 & $\mathrm{e}$ & 0.99 & 0.92 & 0.39 & 0.88 & 0.26 & "wire mesh" & \\
\hline \multicolumn{9}{|c|}{ holes in o-IDTBR } \\
\hline o-IDTBR & $\mathrm{h}$ & 0.41 & 0.17 & 0.06 & 0.14 & 0.04 & & \\
\hline o-IDTBR (exp.geom.) & $\mathrm{h}$ & 0.32 & 0.14 & 0.03 & 0.13 & 0.05 & & \\
\hline
\end{tabular}




\section{References}

1. Frisch, M. J. et al. Gaussian 16 Rev. A.03. (2016).

2. Kresse, G. \& Furthmüller, J. Efficient iterative schemes for ab initio total-energy calculations using a plane-wave basis set. Phys. Rev. B 54, 11169-11186 (1996).

3. Grimme, S., Antony, J., Ehrlich, S. \& Krieg, H. A consistent and accurate ab initio parametrization of density functional dispersion correction (DFT-D) for the 94 elements H-Pu. J. Chem. Phys. 132, 154104 (2010).

4. Tukachev, N. V., Maslennikov, D. R., Sosorev, A. Yu., Tretiak, S. \& Zhugayevych, A. Ground-State Geometry and Vibrations of Polyphenylenevinylene Oligomers. J. Phys. Chem. Lett. 10, 3232-3239 (2019).

5. Zhugayevych, A., Mazaleva, O., Naumov, A. \& Tretiak, S. Lowest-Energy Crystalline Polymorphs of P3HT. J. Phys. Chem. C 122, 9141-9151 (2018).

6. Zhugayevych, A., Postupna, O., Wang, H.-L. \& Tretiak, S. Modification of optoelectronic properties of conjugated oligomers due to donor/acceptor functionalization: DFT study. Chem. Phys. 481, 133143 (2016).

7. Perdew, J. P., Burke, K. \& Ernzerhof, M. Generalized Gradient Approximation Made Simple. Phys. Rev. Lett. 77, 3865-3868 (1996).

8. Kresse, G. \& Joubert, D. From ultrasoft pseudopotentials to the projector augmented-wave method. Phys. Rev. B 59, 1758-1775 (1999).

9. Lee, K., Murray, É. D., Kong, L., Lundqvist, B. I. \& Langreth, D. C. Higher-accuracy van der Waals density functional. Phys. Rev. B 82, 081101 (2010).

10. Klimeš, J., Bowler, D. R. \& Michaelides, A. Van der Waals density functionals applied to solids. Phys. Rev. B 83, 195131 (2011).

11. Zhugayevych, A. et al. Ab Initio Study of a Molecular Crystal for Photovoltaics: Light Absorption, Exciton and Charge Carrier Transport. J. Phys. Chem. C 117, 4920-4930 (2013).

12. Van Der Poll, T. S. et al. Polymorphism of crystalline molecular donors for solution-processed organic photovoltaics. J. Phys. Chem. Lett. 5, 2700-2704 (2014).

13. Coughlin, J. E. et al. A Combined Experimental and Theoretical Study of Conformational Preferences of Molecular Semiconductors. J. Phys. Chem. C 118, 140627161810001 (2014).

14. Lai, H. et al. 3D Interpenetrating Network for High-Performance Nonfullerene Acceptors via Asymmetric Chlorine Substitution. J. Phys. Chem. Lett. 10, 4737-4743 (2019).

15. Zhugayevych, A. \& Tretiak, S. Theoretical Description of Structural and Electronic Properties of Organic Photovoltaic Materials. Annu. Rev. Phys. Chem. 66, 305-330 (2015).

16. Coughlin, J. E., Zhugayevych, A., Wang, M., Bazan, G. C. \& Tretiak, S. Charge delocalization characteristics of regioregular high mobility polymers. Chem. Sci. 8, 1146-1151 (2017). 